\title{
Placental surface shape, function, and effects of maternal and fetal vascular pathology
}

\author{
Carolyn M. Salafia ${ }^{1,2}$, Michael Yampolsky ${ }^{3}$, Dawn P Misra ${ }^{4}$, Oleksander Shlakhter ${ }^{5}$, Danielle \\ Haas $^{1}$, Barbara Eucker 6 , and John Thorp 6 \\ ${ }^{1}$ Placental Analytics, LLC, Larchmont, NY, USA \\ ${ }^{2}$ Department of Obstetrics and Gynecology and Pediatrics, New York Methodist Hospital, Brooklyn, \\ NY \\ ${ }^{3}$ Department of Mathematics, University of Toronto, 40 St. George St, Toronto, Ontario, Canada, \\ M5S2E4 \\ ${ }^{4}$ Department of Family Medicine and Public Health Sciences, Wayne State University School of \\ Medicine.101 E. Alexandrine, Room \#203, Detroit, MI 48201 \\ ${ }^{5}$ Rotman School of Management, University of Toronto, 105 St. George Street, Toronto, ON, M5S \\ $3 \mathrm{E} 6$ \\ ${ }^{6}$ Department of Obstetrics and Gynecology, University of North Carolina at Chapel Hill, Chapel Hill, \\ NC 27599
}

\begin{abstract}
Goal-In clinical practice, variability of placental surface shape is common. We measure the average placental shape in a birth cohort and the effect deviations from the average have on placental functional efficiency. We test whether altered placental shape improves the specificity of histopathology diagnoses of maternal uteroplacental and feto-placental vascular pathology for clinical outcomes.
\end{abstract}

Materials and Methods-1225 placentas from a prospective cohort had chorionic plate digital photographs with perimeters marked at $1-2 \mathrm{~cm}$ intervals. After exclusions of preterm $(\mathrm{n}=202)$ and velamentous cord insertion $(n=44), 979(95.7 \%)$ placentas were analyzed. Median shape and mean perimeter were estimated. The relationship of fetal and placental weight was used as an index of placental efficiency termed " $\beta$ ". The principal placental histopathology diagnoses of maternal uteroplacental and fetoplacental vascular pathologies were coded by review of individual lesion scores. Acute fetal inflammation was scored as a"negative control" pathology not expected to affect shape. ANOVA with Bonferroni tests for subgroup comparisons were used.

Results-The mean placental chorionic shape at term was round with a radius estimated at $9.1 \mathrm{~cm}$. Increased variability of the placental shape was associated with lower placental functional efficiency. After stratifying on placental shape, the presence of either maternal uteroplacental or fetoplacental

\footnotetext{
(C) 2010 Elsevier Ltd. All rights reserved.

Corresponding author: Carolyn M Salafia MD MS, Placental Analytics, LLC, 93 Colonial Avenue, Larchmont NY 10538, Carolyn.salafia@gmail.com.

Publisher's Disclaimer: This is a PDF file of an unedited manuscript that has been accepted for publication. As a service to our customers we are providing this early version of the manuscript. The manuscript will undergo copyediting, typesetting, and review of the resulting proof before it is published in its final citable form. Please note that during the production process errors may be discovered which could affect the content, and all legal disclaimers that apply to the journal pertain.
} 
vascular pathology was significantly associated with lower placental efficiency only when shape was abnormal.

Conclusions-Quantifying abnormality of placental shape is a meaningful clinical tool. Abnormal shapes are associated with reduced placental efficiency. We hypothesize that such shapes reflect deformations of placental vascular architecture, and that an abnormal placental shape serves as a marker of maternal uteroplacental and/or fetoplacental vascular pathology of sufficiently long standing to impact placental (and by extension, potentially fetal) development.

\section{Introduction}

The chorionic plate of the human placenta is commonly depicted as round, with the umbilical cord inserted roughly at the center [1]. However, in clinical practice, the shape of the chorionic disk is rarely truly circular; its shape commonly varies, from round to oval, bi- or multi- lobate, or otherwise irregular. The shape of the placenta is thought to be influenced by where it is implanted in the uterus, regional variations in the decidua (that may determine areas of atrophy), variations in maternal vascular supply (with placental infarcts resulting in altered shape) and perhaps even the "manner of its original implantation" [1]. Benirschke states that "the mechanism by which [non-round shapes] develop is unclear", although "much supports the notion of a secondary conversion from more normal placentation" [1]. In a recent paper [2], we have related this variability in chorionic plate shape to the structure of the underlying placental vascular tree. We can reliably produce multilobate and regularly irregular shapes by changing a parameter that negatively affected the angiogenesis of the model placental vasculature. Furthermore, our empirical results [2] suggest that the earlier in gestation a placental pathology begins, the more likely it is that the effect on placental vascular branching growth will result in a distortion of the placental shape.

In view of the connection between the shape of the chorionic plate and the structure of the chorionic villous tree, it is particularly important to understand what a normal placental shape is and how to measure deviations from the norm. In this paper we use several different techniques to describe an average surface shape for placentas delivered at term. We develop several specific measurements of the deviation from the average which are both practical to implement clinically, and statistically reliable. We further investigate how a deviation from the average placental shape affects the functional efficiency of the placenta, in terms of the birth weight of the baby for a given placental weight.

We then proceed to apply our techniques as a diagnostic tool for the two principal types of vascular pathology that have been described in the placenta, maternal uteroplacental and fetoplacental vascular pathology. Placental histopathology diagnoses have been used by clinicians to diagnose these disorders for almost 50 years since the seminal protocol published by Dr. Kurt Benirschke [3]. Refinements have been made, especially in the recognition of a broad range of fetoplacental and uteroplacental vascular lesions e.g. [4], and in attempts to standardize diagnostic criteria [5-7].

Based on the findings of [2], we have hypothesized that either fetoplacental or maternal uteroplacental vascular pathology that is sufficient to alter placental chorionic plate shape will affect placental function. We predict that fetoplacental or maternal uteroplacental vascular pathology features will have a measureable effect on placental function only when placental chorionic surface shape is abnormal. To test this hypothesis, we have compared the effects of maternal-uteroplacental or fetoplacental vascular pathology on placental functional efficiency in cases with round as compared to irregular shaped chorionic plates. 


\section{Materials and Methods}

\section{Placental Cohort}

The Pregnancy, Infection, and Nutrition Study is a cohort study of pregnant women recruited at mid pregnancy from an academic health center in central North Carolina. Our study population and recruitment techniques are described in detail elsewhere [11]. Briefly, beginning in March 2002, all women recruited into the Pregnancy, Infection, and Nutrition Study were requested to consent to a detailed placental examination. As of October 1, 2004, 94.6 percent of women consented to such examination. Of those women who consented, 87 percent had placentas collected and photographed for image analysis. Of the 1,225 consecutive placentas collected, 202 were delivered pre-term and were excluded. An additional 44 cases were excluded from the present manuscript because the umbilical cord insertion was velamentous; this was not amenable to our mathematical methods which assume that the cord was inserted on the chorionic plate, to be able to center the placenta on the cord insertion point. This left $\mathrm{N}=979$ cases for analysis.

Placental gross examinations, histology review, and image analyses were performed at EarlyPath Clinical and Research Diagnostics, a New York State-licensed histopathology facility under the direct supervision of Dr. Salafia. The institutional review board from the University of North Carolina at Chapel Hill approved this protocol.

The fetal surface of the placenta was wiped dry and placed on a clean surface after which the extraplacental membranes and umbilical cord were trimmed from the placenta. The fetal surface was photographed with the Lab ID number and $3 \mathrm{~cm}$. of a plastic ruler in the field of view using a standard high-resolution digital camera (minimum image size 2.3 megapixels). A trained observer (D.H.) captured series of $x, y$ coordinates that marked the site of the umbilical cord insertion and the perimeter of the fetal surface. The perimeter coordinates were captured at intervals of between $1 \mathrm{~cm}$ and $2 \mathrm{~cm}$, and more coordinates were captured if it appeared essential to accurately capturing the shape of the fetal surface.

\section{Software}

Numerical calculations were carried out using mean-placenta, a Unix-based, ANSI C, software package developed under the terms of the GNU General Public License as published by Free Software Foundation. For visualizations we have used PovRay: a freeware ray tracing program available for a variety of computer platforms; and Maplesoft Maple 9.0 Math \& Engineering software.

\section{Placental histopathology diagnoses}

Placental disease was characterized by gross and microscopic examination of the placenta and decidua. Histology samples were taken from a minimum of four independent sites in the placental parenchyma; diagnoses were assigned to 1 of 3 categories as outlined by Salafia et al. [12-14].

Fetal inflammatory response in umbilical cord and chorionic plate vessels (as a "negative control) and maternal uteroplacental and/or feto-placental vascular lesions were recorded. For the purposes of this study, a fetal inflammatory response was considered present when there was any fetal inflammatory response (ie, umbilical vasculitis, funisitis, or fetal chorionic vasculitis); these lesions have been shown to be present in $<1 \%$ of uncomplicated term births. Maternal uteroplacental vascular pathology was diagnosed in cases by 2 routes: (1) all placentas with at least 1 nonmarginal placental infarct ( $>2 \mathrm{cmfrom}$ the nearest margin) $>1 \mathrm{~cm}^{3}$ in volume and (2) cases with summary scores of histologic items of syncytial knotting, syncytial basophilia, villous fibrosis, and excess perivillous fibrin deposition with cytotrophoblast 
proliferation (each scored on a 0-4 scale, as previously described [12-15]) greater than the birth cohort median value of 7 . Fetoplacental vascular lesions included chorionic and fetal stem vessel mural thrombi, the "hemorrhagic endovasculitis" group of lesions, and avascular villi present in clumps of 50 villi. All placentas were examined by a single expert pathologist (C.M.S.), who was blinded to the patient characteristics when reviewing the placental pathologic findings. For purposes of analysis, the two vascular pathologies and fetal acute inflammatory responses were coded as present vs not present.

\section{Calculating the mean shape of the chorionic plate}

A square grid of $500 \times 500$ pixels was superimposed on the images of the placentas in the sample, each of which was rescaled to the actual size as reflected by the ruler in the field of view The size of one pixel was chosen to equal $0.1 \mathrm{~cm}$, so the total coverage of the grid was a square of $50 \times 50 \mathrm{~cm}^{2}$. The insertion point of the umbilical cord was placed at the center of the square, and the point on the placental perimeter closest to the rapture of the amniotic sac was placed on the negative vertical axis, for consistency.

For each of the placentas in the dataset, all the pixels inside of the placental perimeter, including those intersecting with the boundary, were marked. Thus, for each placenta, an approximate area covered by it was obtained as the union of the marked pixels. For the $n$-th placenta in the dataset, we denote $S_{n}$ the region of the $50 \times 50$-square, which is covered by its surface. $W_{n}$ denotes the union of the marked pixels for this placenta, so that the edge of $W_{n}$ is no more than the size of one pixel $(0.1 \mathrm{~cm})$ removed from the edge of $S_{\mathrm{n}}$. To calculate the mean shape of the chorionic plate, for each pixel $p(x, y), x=1 \ldots 500, y=1 \ldots 500$ of the grid, we marked the total number $t(x, y)$ of the placentas for which $p(x, y)$ lies in $W_{n}$. The central pixels will thus be covered by all of the placentas, that is $t(x, y) \approx N$ (the total number of placentas in the dataset) for $x, y \approx 250$, and the peripheral ones will be covered by very few.

We let $W_{\text {median }}$ be the union of all pixels $p(x, y)$ with $t(x, y) \geq N / 2$.It is thus the median shape of the chorionic plate in our sample.

\section{Calculating the mean placental perimeter}

To calculate the mean chorionic plate perimeter, the insertion point of the umbilical cord was again placed at the origin, and the point on the perimeter closest to the rupture of the amniotic sac was positioned on the negative vertical axis. The perimeters of the placentas in the dataset were rescaled to the real size. The points in the perimeter were then averaged inside a sector of $18^{\circ}$, thus obtaining 20 radial markers for each placenta (see Figure 2). Each of the markers was then averaged over the whole dataset, thereby giving 20 mean placental radii from the umbilical insertion point spaced at $18^{\circ}$ angular intervals. Averaging these 20 mean radii we obtain the mean placental radius $R_{\text {mean }}$ from which the perimeter is readily calculated.

\section{Measuring the deviation of the placental perimeter from the mean}

We have used two measurements of the deviation from the mean placental shape. The first one, which we call sigma, is the mean square deviation of the 20 perimeter radial markers from the mean radius $R_{\text {mean }}$. The second one is based on the difference between the median placental shape $W_{\text {median }}$ and the pixellated chorionic plate shape $W_{n}$ of the $n$-th placenta in our dataset. We add up the area of all the pixels which are covered by one of the shapes $W_{\text {median }}, W_{n}$ but not by both of them at the same time. We call this total area the symmetric difference between the placental shape and the mean. 


\section{Measuring placental eccentricity}

As a measure of placental eccentricity, for each placenta in the dataset we found the farthest and closest of the 20 radial markers, and calculated the ratio of the distances to them from the umbilical insertion point: $E_{n}=D_{\max } / D_{\min }$. For a round placenta with centrally inserted umbilical cord, the value of eccentricity is 1 .

\section{Placental Functional Efficiency $(\beta)$}

We have previously shown that placental weight is proportional to birth weight to the $3 / 4$ power $[8,9]$. We suggested that such fractional scaling is due to the fractal structure of the placental vasculature, and introduced a scaling exponent describing placental functional efficiency (in terms of birth weight yielded per gram of placental weight). This scaling exponent, which we termed $\beta$, is defined as the ratio of the logs of placental weight and birth weight $(\beta=\log$ PW/ $\log \mathrm{BW}$ ) [8,9]. Thus, as $\beta$ increases, the placental size is greater relative to birth weight (implying reduced placental functional efficiency), and as $\beta$ decreases, the birth weight is relatively larger for the given placental weight (indicating greater placental efficiency).

\section{Statistical analysis}

Contingency tables compared categorical variables. Sigma, symmetric difference, and beta are each normally distributed. ANOVA was used to test for differences between histopathology diagnostic categories and the continuous outcomes. Pearson correlations tested for relationships between continuous variables. Multivariate comparisons were performed using linear regression.

\section{Results}

\section{Mean placental shape}

The median chorionic plate shape is round, as seen in Figure 1. We calculated the mean radius of a pixel on the boundary of $W_{\text {median }}$ from the umbilical insertion point as $9.066 \mathrm{~cm}$ (range $8.76-9.46 \mathrm{~cm}$, mean square deviation $0.181 \mathrm{~cm}$, or $2 \%$ ). We have also calculated the shapes $W_{40 \%}$ and $W_{30 \%}$ which correspond to the values of $t(x, y)$ greater than $0.4 \mathrm{~N}$ and $0.3 \mathrm{~N}$, respectively. They are also round (Figure 1).

Averaging of the 20 placental radial markers is illustrated in Figure 2. This procedure also yields a round shape with mean average chorionic plate radius $R_{\text {mean }}=9.01 \mathrm{~cm}$. The mean value for each of the 20 marked radii were within $3.3 \%$ of the average chorionic plate radius (range $8.71-9.27 \mathrm{~cm}$, and mean square deviation $(0.18 \mathrm{~cm}$ or $1.9 \%)$.

We have calculated the average area of the region covered by the chorionic plate shape as the arithmetic mean of chorionic plate area as $A_{\text {mean }}=285.58 \mathrm{~cm}^{2}$, standard deviation of $60.78 \mathrm{~cm}^{2}$ or $21.2 \%$, range $135.03 \mathrm{~cm}^{2}$ and the maximal value $649.44 \mathrm{~cm}^{2}$. While the standard deviation appears large, recall that for a disk of radius $R$, the change of radius by $\Delta R$ changes the area by approximately $2 \pi \cdot \Delta R$. Thus even if our placentas were all round, a $21.2 \%$ variance of the area would correspond to less than $3.4 \%$ of radial variance. Note that $A_{\text {mean }}$ is the area of a disk with radius $R_{\text {are }}=9.53 \mathrm{~cm}$, which can viewed as another measurement of the mean placental radius.

We have calculated the average radius $R_{n}$ of a chorionic plate in our dataset by averaging the 20 radial markers obtained as described above. The standard deviation of $R_{n}$ from the mean value $R_{\text {mean }}=9.01 \mathrm{~cm}$ is $1.07 \mathrm{~cm}$, or $11.8 \%$. Thus both the chorionic plate area, and the outer radius from the umbilical insertion point have tight distributions with narrow standard deviations. The $95 \%$ confidence intervals for chorionic plate area and chorionic plate radius are $\left[281.77 \mathrm{~cm}^{2}, 289.38 \mathrm{~cm}^{2}\right]$ and $[8.94 \mathrm{~cm}, 9.07 \mathrm{~cm}]$ respectively. 


\section{Average placental eccentricity}

Mean value of placental eccentricity in our birth cohort is $E_{\text {mean }}=1.68$; median value is $E_{\text {median }}=1.49$. This indicates that deviations from the mean round shape are common for placentas in our dataset.

\section{Placental histology features}

As noted above in Methods, the principal pathology (most prominent pathology process) was used as the key variable in analysis with the 463 (47\% of the total 979 case) cases with any of the histopathology types as defined above. Acute inflammation was considered the "principal" pathology in 83 cases; maternal uteroplacental vascular pathology in 167 cases and fetoplacental vascular pathology in 134 cases Of these 62 had both maternal uteroplacental and fetoplacental vascular pathology.

\section{Histopathology diagnoses and placental shape (deviation measures)}

No pathology diagnosis was associated with either sigma or with symmetric difference (all $\mathrm{p}>0.10)$.

\section{Histopathology diagnoses and placental functional efficiency $(\beta)$}

Vascular pathology was correlated with increased $\beta(\mathrm{p}<0.0001)$, specifically, maternal uteroplacental vascular pathology (absent $0.748 \pm 0.020 \mathrm{v}$. present $0.754 \pm 0.023, \mathrm{p}=0.002$ ) and fetoplacental vascular pathology (absent $0.748 \pm 0.020 \mathrm{v}$. present $0.755 \pm 0.022, \mathrm{p}=0.001$ ). The combined presence of both maternal uteroplacental and fetoplacental vascular pathologies did not have a greater effect on $\beta(0.754+0.022)$ than when each pathology was analyzed alone. Acute inflammation was not associated with a difference in $\beta$.

\section{Placental shape and placental functional efficiency $(\beta)$}

Sigma and symmetric difference were both significantly correlated with $\beta$, with $\rho=0.138$ and 0.209 respectively, consistent with previously reported results in which we used an alternative method for analysis of irregularity of placental shape $[9,10]$.

\section{Histopathology diagnosis associations with placental functional efficiency: Modification by placental shape (Table 1)}

Stratifying the cohort into those with sigma less or greater than the mean (regular versus more irregular shapes), maternal uteroplacental and fetal fetoplacental vascular pathology were correlated with an increased $\beta$ only in the cases with greater sigma (more irregular shape, $p=0.006$ and $p=0.004$, respectively). Finally, consistent with the unstratified analyses, acute inflammation was not associated, in either direction, with $\beta$ regardless of placental shape.

\section{Discussion}

In previous work [2], we have shown that the placental chorionic surface shape closely mirrors the pattern of chorionic vascular growth. Burgeoning ultrasonography literature has linked various detectable markers of atypical placental shape to later significant fetal morbidity and/ or mortality, making quantification of placental shape imperative.

Our measurements demonstrate that the average placental shape is a disk centered at the umbilical insertion point. This may have been conventionally believed, yet at a closer examination, several aspects of the results are notable. Firstly, very few placentas appear truly round with a centrally inserted umbilical cord. Variability of shape is the norm. This is manifested in our measurements of mean and median placental eccentricity, which are 1.68 and 1.49 respectively, reflecting a typically significant deviation from a round placental shape 
with a centrally inserted umbilical cord. Yet, these deviations are equally likely to happen in every direction, so they cancel each other in the average. This is confirmed by observing the shapes $W_{40 \%}$ and $W_{30 \%}$, which average large deviations from the mean (Figure 1). To make these observations, it is of a vital importance that placental surface pictures in the dataset have been given a fixed, biologically consistent orientation - and thus no bias has been introduced into the directionality of perturbations of the shape.

The second important point is the impressive agreement of the different methods of measurement which we have used on what the average placental shape is: a disk centered at the umbilical insertion point with radius of about $9 \mathrm{~cm}$. This, and tight confidence intervals for the measurements, imply that quantifying the abnormality of the placental shape is meaningful in practice, and is little affected by the technique chosen.

We see a significant correlation between large deviations of the placental shape from the mean and reduced placental efficiency, quantified as a larger scaling exponent $\beta$ (smaller birth weight for the given placental weight). This confirms the hypothesis we developed in $[2,8,9]$ : an abnormal placental shape is associated with an altered placental vascular architecture, which in turn is associated with a reduced functional efficiency.

Turning to the specific diagnostic value of measuring the placental surface shape, we have demonstrated its utility in improving the specificity of placental histology diagnoses for clinical outcomes. In this study, maternal uteroplacental and fetoplacental vasculopathies only affected $\beta$ (measure of placental functional efficiency) when placental shape was irregular, consistent with perturbation of placental vascular growth at point(s) across gestation [2].

Our results can thus be interpreted as showing that deviations of the placental shape from the mean marks an adverse effect of either maternal uteroplacental vascular pathology or fetoplacental vascular pathology on the fetoplacental development, as measured by placental functional efficiency $(\beta)$. In combination with our empirical growth model of the placental vasculature [2], these data suggest that the combination of histopathology diagnosis of either maternal uteroplacental or fetoplacental vascular pathology with greater shape deviation represents either a longer standing disease process, a more severe disease process, or both. Adding shape deviation to the clinical diagnostic tool kit should improve diagnostic specificity and clinical understanding.

Acute intraamniotic inflammations were not associated with either abnormal placental efficiency or abnormal shape. These processes are generally believed to arise close to delivery, being analyzed in relation to factors that are counted in minutes or hours [e.g., 16,17]. In agreement with our DLA model of placental vascular growth [2], they appear to be of an insufficiently long duration to have any measurable effect on the placental vascular growth, and hence placental shape.

Limitations of our work are two-fold. The first is computational. We excluded velamentous umbilical cords as the necessary measurements could not be made and therefore we were missing crucial data to compute area and radius. We would speculate that these most asymmetric examples of placental growth would be on the extreme end of the continuum we have analyzed moving from centrally sited cords to more and more peripherally located cords. However, our mathematical methods require centering the placental image on the umbilical cord insertion point. Secondly, histopathology diagnoses remain subjective with problematic interrater reliability. While a single rater performed these assessments blinded to all data except gestational age, we recognize that these diagnoses might not be duplicated by a second reviewer. We maximized potential interrater reliability by dichotomizing the measures as others have done [5-7]. However, the relatively new capacity to digitize an entire histology slide, making it amenable to image analysis techniques, offers the potential for automated, 
reliable and more quantitative measures. We have currently demonstrated that such an approach is amenable to diagnoses of acute inflammation [18], and expect that other diagnostic pathologies will follow.

\section{Conclusions}

Different mathematical approaches estimated that the mean placental surface shape is a disk centered at the umbilical insertion point with radius of $\sim 9 \mathrm{~cm}$. The confidence limits provided herein can be useful clinical tools in determining what are the key diagnostic elements of placental pathology, both gross and histologic, that affect placental function.

Combined with a simply calculated measure of abnormal placental shape, the impact of vascular pathology (either maternal uteroplacental or fetoplacental) on placental function depended on placental shape. A burgeoning ultrasonography literature has linked various detectable markers of atypical placental shape to later significant fetal morbidity and/or mortality, thus making a deeper understanding of the genesis of placental shape imperative.

\section{Acknowledgments}

This work was partially supported by NSERC Discovery Grant (M. Yampolsky), by NARSAD Young Investigator Award (C. Salafia), by K23 MidCareer Development Award NIMH K23MH06785 (C. Salafia).

\section{References}

1. Benirschke, K.; Kaufmann, P.; Baergen, R. Pathology of the Human Placenta. 5th ed.. Vol. Chapter 7. New York, New York: Springer Verlag; 2006. Architecture of Normal Villous Trees; p. 121-159.

2. Yampolsky M, Salafia CM, Shlakhter O, Haas D, Eucker B, Thorp J. Modeling the variability of shapes of a human placenta. Placenta 2008;29:790-797. [PubMed: 18674815]

3. Benirschke K. Examination of the placenta. Prepared for the Collaborative Study on Cerebral Palsy, Mental retardation and other Neurological and Sensory Disorders of Infancy and Childhood, National Institute of Neurological Diseases and Blindness, US Department of Health, Education and Welfare, Public Health Service. 1961

4. Sander CM, Gilliland D, Akers C, McGrath A, Bismar TA, Swart-Hills LA. Livebirths with placental hemorrhagic endovasculitis: interlesional relationships and perinatal outcomes. Arch Pathol Lab Med 2002;126(2):157-164. [PubMed: 11825110]

5. Redline RW, Faye-Petersen O, Heller D, Qureshi F, Savell V, Vogler C. Society for Pediatric Pathology, Perinatal Section, Amniotic Fluid Infection Nosology Committee. Amniotic infection syndrome: nosology and reproducibility of placental reaction patterns. Pediatr Dev Pathol 2003;6(5): 435-448. [PubMed: 14708737]

6. Redline RW, Boyd T, Campbell V, Hyde S, Kaplan C, Khong TY, Prashner HR, Waters BL. Society for Pediatric Pathology, Perinatal Section, Maternal Vascular Perfusion Nosology Committee. Maternal vascular underperfusion: nosology and reproducibility of placental reaction patterns. Pediatr Dev Pathol 2004;7(3):237-249. [PubMed: 15022063]

7. Redline RW, Ariel I, Baergen RN, Desa DJ, Kraus FT, Roberts DJ, Sander CM. Fetal vascular obstructive lesions: nosology and reproducibility of placental reaction patterns. Pediatr Dev Pathol 2004;7(5):443-452. [PubMed: 15547768]

8. Salafia CM, Misra DP, Yampolsky M, Charles AK, Miller RK. Allometric metabolic scaling and fetal and placental weight. Placenta 2009;30(4):355-360. [PubMed: 19264357]

9. Salafia CM, Yampolsky M. Metabolic scaling law for fetus and placenta. Placenta 2009;30(5):468471. [PubMed: 19285342]

10. Yampolsky M, Salafia CM, Shlakhter O, Haas D, Eucker B, Thorp J. Centrality of the umbilical cord insertion in a human placenta influences the placental efficiency. Placenta 2009;30(12):1058-1064. [PubMed: 19879649] 
11. Savitz DA, Dole N, Williams J, et al. Determinants of participation in an epidemiological study of preterm delivery. Paediatr Perinat Epidemiol 1999;13:114-125. [PubMed: 9987790]

12. Salafia CM, Vogel CA, Bantham KF, Vintzileos AM, Pezzullo J, Silberman L. Preterm delivery: Correlations of fetal growth and placental pathology. Am J Perinatol 1992;9:190-193. [PubMed: 1575840]

13. Salafia CM, López-Zeno JA, Sherer DM, Whittington SS, Minior VK, Vintzileos AM. Histologic evidence of old intrauterine bleeding is more frequent in prematurity. Am J Obstet Gynecol 1995;173:1065-1070. [PubMed: 7485294]

14. Salafia CM, Ernst LM, Pezzullo JC, Wolf EJ, Rosenkrantz TS, Vintzileos AM. The very low birthweight infant: Maternal complications leading to preterm birth, placental lesions, and intrauterine growth. Am J Perinatol 1995;12:106-110. [PubMed: 7779189]

15. Baker AM, Braun JM, Salafia CM, Herring AH, Daniels J, Rankins N, Thorp JM. Risk factors for uteroplacental vascular compromise and inflammation. Am J Obstet Gynecol 2008 Sep;199(3) 256.e1-9.

16. Park HS, Romero R, Lee SM, Park CW, Jun JK, Yoon BH. Histologic Chorioamnionitis is More Common after Spontaneous Labor than after Induced Labor at Term. Placenta. 2010 Jul 21; [Epub ahead of print.].

17. Rouse DJ, Weiner SJ, Bloom SL, Varner MW, Spong CY, Ramin SM, Caritis SN, Peaceman AM, Sorokin Y, Sciscione A, Carpenter MW, Mercer BM, Thorp JM Jr, Malone FD, Harper M, Iams JD, Anderson GD. Eunice Kennedy Shriver National Institute of Child Health and Human Development Maternal-Fetal Medicine Units Network. Second-stage labor duration in nulliparous women: relationship to maternal and perinatal outcomes. Am J Obstet Gynecol 2009 Oct;201(4) 357.e1-7.

18. Thomas, KA.; Sottile, MJ.; Salafia, CM. Unsupervised Segmentation for Inflammation Detection in Histopathology Images. In: Elmoataz, A., et al., editors. ICISP 2010, LNCS 6134. 2010. p. 541-549. 


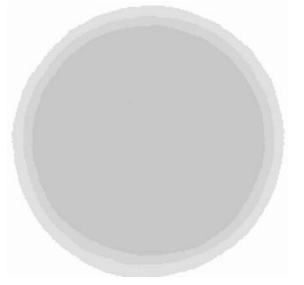

Figure 1.

Median placental shape $W_{\text {median }}$ (smallest disk) and $W_{40 \%}, W_{30 \%}$ (two larger disks). The position of the insertion point of the umbilical cord is in the center of the picture. 


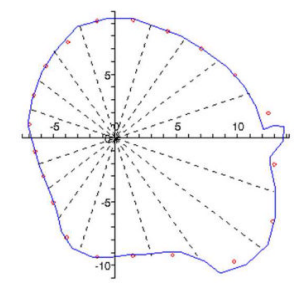

Figure 2.

Calculation of the 20 radial markers for one of the placentas in the dataset. The solid contour marks the perimeter of the placental surface. The 20 radial sectors emanate from the umbilical insertion point. Each marker is indicated with a small circle. It averages the distance from the perimeter curve to the insertion point within the sector. 


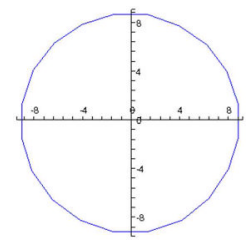

Figure 3.

The positions of the 20 mean perimeter markers, connected to show the mean placental perimeter. The umbilical insertion point is at the origin. 

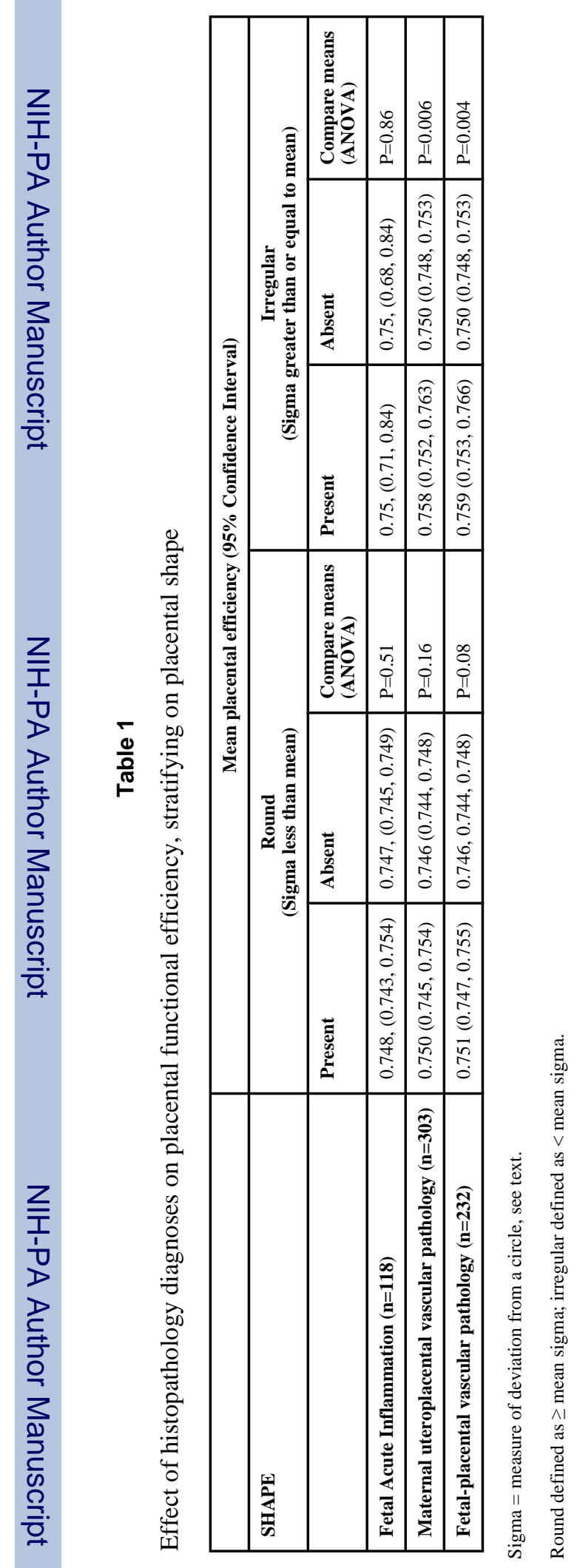

Placenta. Author manuscript; available in PMC 2011 November 1. 